\title{
Micromachined Silicon Cantilevers and Tips for Scanning Probe Microscopy
}

\author{
R.A. Buser, J. Brugger and N.F. de Rooij \\ Institute of Microtechnology, University of Neuchâtel, Breguet 2, CH-2000 NEUCHATEL \\ Switzerland
}

\begin{abstract}
A monocrystalline silicon lever with an integrated silicon tip for a Force/Friction Microscope was realized. Theoretical studies have been carried out to find the shape and dimensioning according to the mechanical system requirements. Moreover, sharp tips with a high aspect ratio could be demonstrated.
\end{abstract}

\section{INTRODUCTION}

Scanning Tunneling Microscopes and Atomic Force Microscopes, known under the expression Scanning Probe Microscope (SPM), are meanwhile well established methods to analyze surfaces and the principle of their operation is published widely12,3. In AFM a point probe reacts mechanically on interatomic forces of the sample and generates a topographic view of the surface with an atomic resolution. The mechanical part typically consists of a cantilever as a spring element with a sharp tip at its end. The advantage of using thin films to form cantilevers for SPM is the possible high aspect ratio with a high stiffness in $\mathrm{x}$ and $\mathrm{y}$ direction (in the plane) and an adjustable stiffness in z-direction (out of the plane), which does to some degree not influence its resonance frequency. We have fabricated such $\mathrm{SiO}_{2}$ cantilevers ${ }^{4}$, which have been successfully used in SPM applications 2 .

In microelectronics itself such STM/AFM are employed as profilers. Thereby the chip surface is measured in three dimensions, which in the comparison with an ideal chip allows a control of quality and even the localization of defects. For this application it would be very important to have arrays, which is only possible with batch fabricated heads.

Seeing single atoms opens the possibility to observe processes on atomic level and to utilize an altering of the constellation as storage device or as surface preparation for catalytic purposes. First steps of this engineering on a atomic level were already undertaken with tunnel microscopes ${ }^{5}$.

\section{FABRICATION OF BIDIRECTIONAL CANTILEVERS}

In the field of AFM, some interest has arisen to measure not only the force in the z-direction but also parallel to the scanning direction (say y-direction) especially to study tribological behaviour of surfaces. This implies that the stiffness in $z$ and $y$-direction should become similar. For a micromachined cantilever, one with a square cross section would be ideal. This is no longer achievable with thin film cantilevers since the width of the beam should then become smaller than $1 \mu \mathrm{m}$, an unrealistic approach for the application in mind. Theoretical studies have been carried out to find the shape and dimensioning according to the mechanical system requirements, i.e. a force constant $\mathrm{k}<1 \mathrm{~N} / \mathrm{m}$ for an accurate force detection, a resonance frequency $>10 \mathrm{kHz}$ to be able to filter out vibrational noise and a high force constant in $\mathrm{x}$ 
direction. In order to find a compact shape with feasible dimensions, we investigated analytically a simple beam and different folded structures.

A meander type cantilevers allows to shorten the overall length of the cantilever up to half of the length of the simple beam with the same cross section and force constant. Also, it is possible to adjust the ratio of the stiffness in $y$ and $z$ direction to a certain degree. By the simulation program ANSYS(C) ${ }^{6}$ we optimized the shape with the following results:Force constants $[\mathrm{N} / \mathrm{m}] \mathrm{k}_{\mathrm{z}}=0.79 \mathrm{k}_{\mathrm{y}}=1.02 \mathrm{k}_{\mathrm{x}}=20.4$ and the resonance frequencies $[\mathrm{kHz}] \mathrm{f}_{\mathrm{z}}=12$, $f_{y}=14, f_{z}=29$. For a force at the end of the cantilever of $10 \mu \mathrm{N}$ we found that the maximal stress at the clamping $\left(\sigma_{\max }=15^{*} 10^{6} \mathrm{~N} / \mathrm{m}^{2}\right)$ is about hundred times below the fracture stress. Silicon is by the way very well suited for resonance methods because of its very high intrinsic Q-factor ${ }^{7}$.

On a double-side polished $280 \mu \mathrm{m}$ thick $<100>$ wafer, a $1.5 \mu \mathrm{m}$ thermal $\mathrm{SiO}_{2}$ was grown. On the backside windows were opened for the formation of a membrane $(\approx 15 \mu \mathrm{m}$ thick). This was obtained by anisotropic etching with $40 \% \mathrm{KOH}$ at $60^{\circ} \mathrm{C}$ from the backside while the topside was protected by a mechanical chuck. On this membrane the cantilever was patterned by means of $6.5 \mu \mathrm{m}$ thick photoresist. Anisotropic Reactive Ion Etching (RIE) using a $\mathrm{C}_{2} \mathrm{ClF}_{5} / \mathrm{SF}_{6}$ gas mixture ends in silicon columns of $12 \mu \mathrm{m}$ height, with nearly vertical sidewalls and without under-cutting ${ }^{8}$. We could thus fabricate cantilevers with a quadratic cross section (see Fig.1).

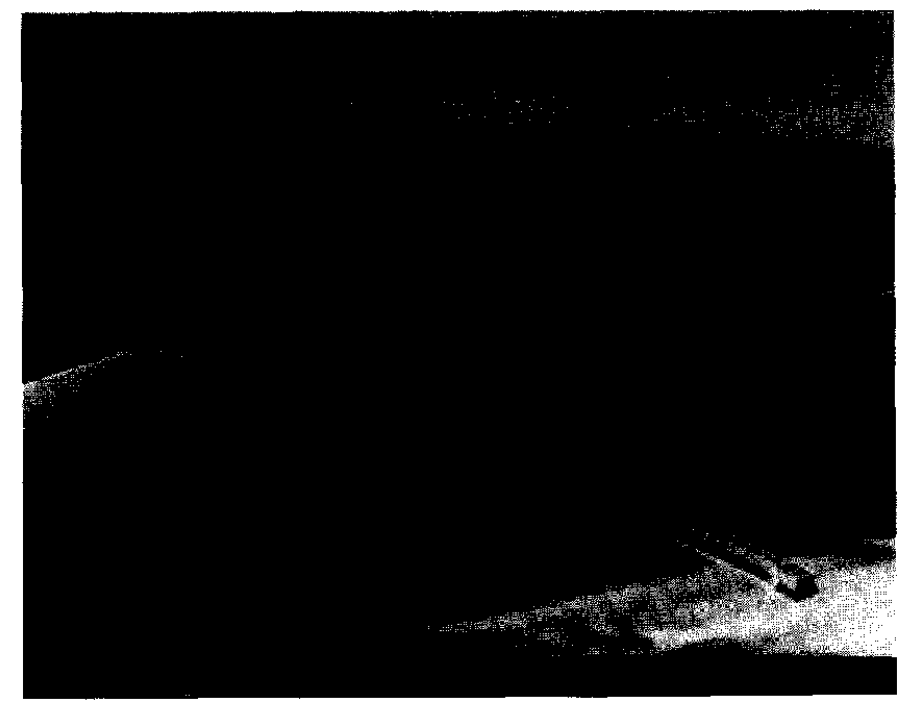

Fig.1. m-Si cantilever with square cross section, designed as a meander shape. The clamping is not at the central line, which improves the decoupling of the y-movement from the $x$ movement.

\section{FABRICATION OF TIPS ON WAFER}

A crucial part of the AFM point probe is the tip, which has to be as sharp as possible. It would be of great benefit to integrate it directly on the cantilever. We investigated methods to form tips in $\mathrm{m}-\mathrm{Si}$, which are compatible with the microfabrication of cantilevers 
By means of two successive photolithographies, we patterned a $10 \mu \mathrm{m}$ square double layer mask consisting of $1.5 \mu \mathrm{m}$ thick silicon dioxide (below) and $6.5 \mu \mathrm{m}$ thick photoresist as shown in Fig.2. Again RIE with $\mathrm{C}_{2} \mathrm{ClF}_{5} / \mathrm{SF}_{6}$ was applied to obtain a column (Fig.2a.), which is then thinned during a $15 \mathrm{~s}$ isotropic acid etch step in $\mathrm{HNO}_{3}: \mathrm{HF}: \mathrm{CH}_{3} \mathrm{COOH}$ (Fig.2b). The silicon dioxide cap, previously buried under the photoresist layer, functions now as the etch mask and enhances the formation of a sharply pronounced tip as shown in Fig.3.

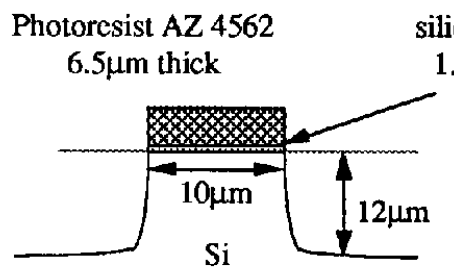

a) silicon dioxide $1.5 \mu \mathrm{m}$ thick

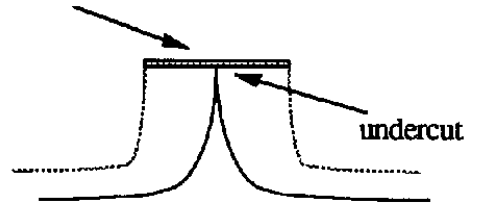

b)

Fig.2. Tip formation exploiting a two stage dry-anisotropic / wet-isotropic etch process. a) 12 $\mu m$ high silicon columns obtained by RIE etching b) Column thinning and pronounced tip formation by wet etching

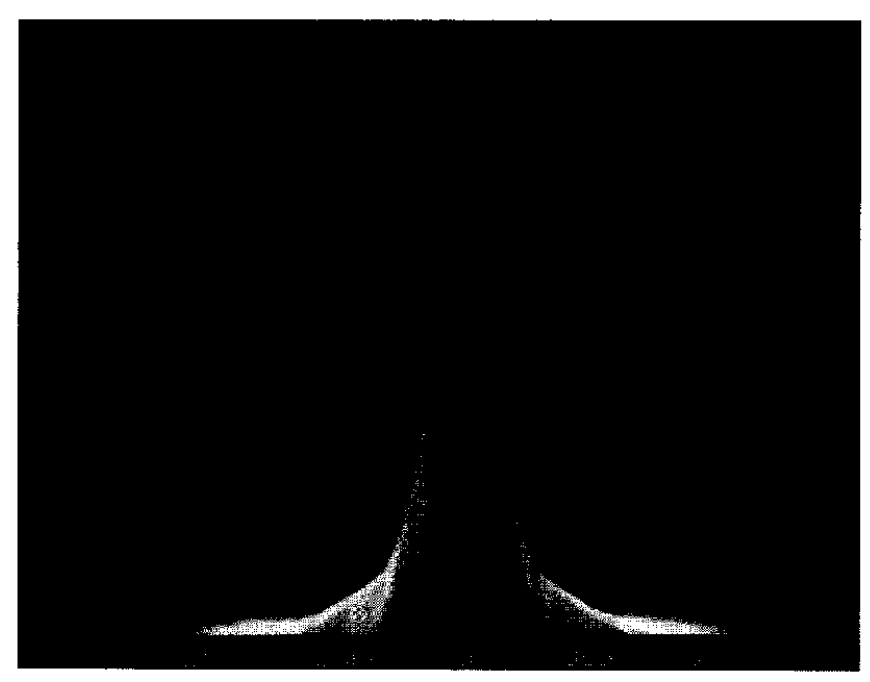

Fig.3. A SEM of a $10 \mu \mathrm{m}$ high tip with a high aspect ratio.

\section{FABRICATION OF TIPS ON CANTILEVER}

The formation of the cantilever was similar to that without tip excepting that simultaneously with the opening of the windows on the backside for the machining of the membrane, at the topside $10 \mu \mathrm{m}$ wide squares were patterned serving as mask for the subsequent tip formation. Moreover the membrane was $25 \mu \mathrm{m}$ instead of $15 \mu \mathrm{m}$, since it will be etched further during the tip formation. The photoresist covered the $\mathrm{SiO}_{2}$ cap and serves as masking for the RIE step.The same $\mathrm{C}_{2} \mathrm{ClF}_{5} / \mathrm{SF}_{6}$ gas mixture was used to etch the $15 \mu \mathrm{m}$ deep vertical sidewalls of the cantilever and to obtain a very thin $(10 \mu \mathrm{m})$ membrane. 
Next an acid etching with a diluted mixture of $\mathrm{HNO}_{3}: \mathrm{HF}: \mathrm{CH}_{3} \mathrm{COOH}$ is carried out during about $2 \mathrm{~min}$. This final etch step is crucial since simultaneously it forms the tip (using the buried oxide cap as mask), pierces through the membrane and consequently releases the silicon spring. Additionally this wet etching smooths the dry etched rough surface and cleans from burned organic photoresist (Fig.4).

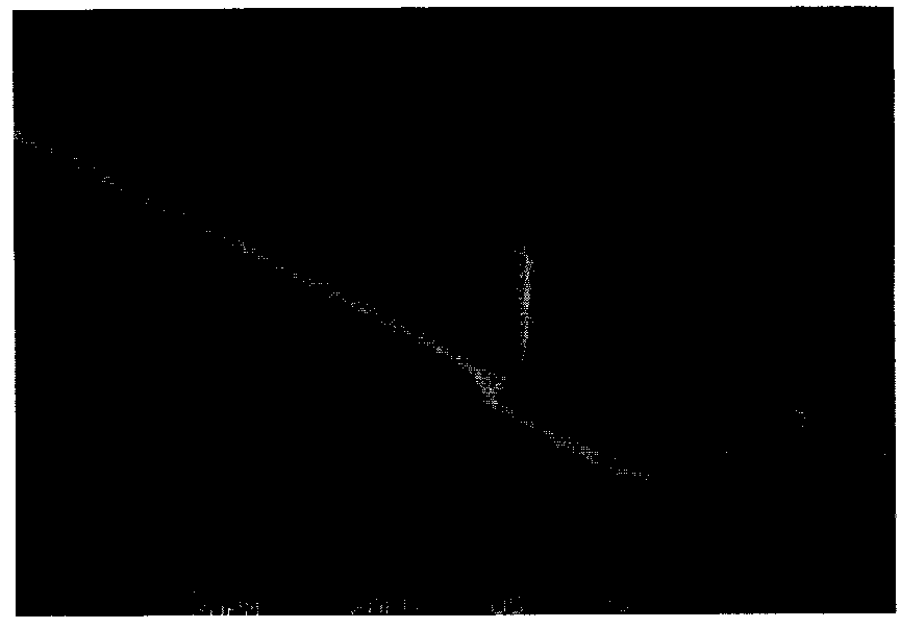

Fig.4. Integrated $m$-Si Tip on top of the cantilever

\section{CONCLUSIONS}

In conclusion, we could show the realization of a monocrystalline silicon lever with an integrated silicon tip for a Force/Friction Microscope. Moreover, sharp tips with a high aspect ratio could be demonstrated. Of course the described method can also be used to fabricate board type cantilevers with an integrated tip, which is even easier than fabricating the tip on a square cross section. For the detection of the displacement we use an optical beam deflection method.

\section{Acknowledgements}

We would like to thank Prof.Güntherodt from the University of Basel and his co-workers for the support of this work and Mr.C.Ketterer for the SEM photographs.

1. K.Wickramasinghe; Scanning probe microscopy: Current status and future trends. J.Vac.Sci. Tcchnol. A 8 (1) Jan/Feb 1990 p. 363.

2. E.Meyer at al.; Comparative Study of Lithium Fluoride and Graphite by Atomic Force Microscopy (AFM, Proceeding of the STM Conference, Oxford, August 1988

3. C.F.Quate; Imaging with the tunneling \& force Microscopes Proceeding of the IEEE Micro Electromechanical Systems, Napa Valley, Cal. 11-14 Feb. 1990, p.188.

4. R.A.Buser; Theoretical and Experimental Investigations on Silicon Single Crystal Resonant Structures, Ph.D. Thesis University of Neuchâtel, IMT, June 1989.

5. U.Staufer et al , Appl. Phys. Lett. 51, 244 (1987)

6. ANSYS is a well known FEM program distributed by Swanson Analysis Systems, Inc. Houston.

7. R.A.Buser, N.F. de Rooij, "Very high Q-factor Resonators in Monocrystalline Silicon, Sensors and Actuators," A21-A23 (1990) pp. 323-327.

8. C.Linder, T.Tschan, and N.F. de Rooij, "Dry Etching Techniques as a New IC Compatible Tool for Silicon Micromachining," presented at Transducers 91. San Francisco. 